\title{
Targeting anesthesia to the pelvic plexus for prostate biopsy
}

Injecting lidocaine proximal to the pelvic plexus provides superior pain relief to the standard periprostatic nerve block procedure. This was the main finding of a prospective randomized trial of 80 men presenting for prostate biopsy to a single center in Turkey.

The study team used color Doppler ultrasonography to locate the pelvic plexus in 40 patients. Lidocaine $(2 \mathrm{ml}$ of a $2 \%$ solution) was injected adjacent to the neurovascular bundle at the apices of the seminal vesicles. In the other 40-strong group, periprostatic nerve block was achieved by administering the same amount of $2 \%$ lidocaine bilaterally between the seminal vesicles and the base of the prostate.

Using a visual analog scale $10 \mathrm{~min}$ after biopsy completion, men in both groups rated the tissue sampling phase (12 cores) as the most painful part of the procedure, followed by injection of anesthetic, then insertion of the transrectal probe. Participants who received a pelvic plexus block reported markedly less pain during injection and sampling than their counterparts in the periprostatic nerve block group.

The authors assert that precise selection of sites for anesthetic injectionfacilitated by Doppler visualization - is crucial to the success of the pelvic plexus approach. Incorporating Doppler ultrasonography into their biopsy regimen prolonged the procedure by less than a minute, and incurred no additional operating costs.

Suzanne J. Farley

Original article Akpinar, H. et al. Doppler ultrasonographyguided pelvic plexus block before systematic needle biopsy of the prostate: a prospective randomized study. Urology 74, 267-271.e1 (2009). 